\title{
Ensayo clínico aleatorizado y controlado para valorar una intervención por una unidad de hospitalización domiciliaria en la reducción de reingresos y muerte en pacientes dados de alta del hospital tras un ingreso por insuficiencia cardiaca
}

\author{
Begoña Aldamiz-Echevarría Iraúrgui, Javier Muñiz, José A Rodríguez-Fernández, \\ Luciano Vidán-Martínez, Miguel Silva-César, Fernando Lamelo-Alfonsín, José L Díaz- \\ Díaz, Vicente Ramos-Polledo, Alfonso Castro-Beiras
}

\begin{abstract}
Resumen
Introducción y objetivos. Evaluar la eficacia de una intervención de educación en pacientes con insuficiencia cardiaca (IC) realizada por hospitalización a domicilio.

Métodos. Ensayo clínico aleatorizado y controlado. Se incluyó a 279 pacientes con diagnóstico clínico de IC dados de alta de un hospital terciario entre febrero de 2001 y junio de 2002. Se excluyó a los pacientes con demencias, enfermedad terminal no cardiológica o enfermedad pulmonar obstructiva crónica. La información recogida incluyó las causas de la descompensación. La intervención fue fundamentalmente de tipo educativo, en el domicilio del participante, y se extendió hasta 15 días después del alta. Se realizaron ajustes de tratamiento durante la primera semana cuando fue necesario. El objetivo principal fue determinar la incidencia acumulada de reingreso o muerte. Los objetivos secundarios fueron la incidencia de reingreso y la mortalidad, así como la utilización de los servicios de urgencia. Se llevó a cabo un seguimiento telefónico a los 3, 6 y 12 meses, y una revisión de las historias clínicas si era necesario. Asimismo, se valoró la utilización de servicios de urgencias los primeros 6 meses.

Resultados. Al año, 62 pacientes de 137 (45,3\%) ingresaron o murieron en el grupo de intervención, en comparación con 75 de $142(52,8 \%)$ en el grupo control $(p=0,232$; riesgo relativo $[R R]=0,86)$. En los pacientes que se descompensaron por incumplimiento terapéutico, 16 de $45(35,6 \%)$ ingresaron o murieron en el grupo de intervención, en comparación con 34 de $56(60,7 \%)$ en el grupo control ( $\mathrm{p}=0,016 ; \mathrm{RR}=0,59)$.

Conclusiones. Esta intervención es factible pero, administrada de manera indiscriminada a todo paciente dado de alta por IC, en el mejor de los casos sólo podemos esperar un beneficio modesto, que en este estudio en particular no llegó a alcanzar significación estadística.
\end{abstract}

\section{Palabras clave}

Insuficiencia cardiaca. Ensayo clínico aleatorizado. Programas de cuidados gestionados. Adhesión del paciente.

\section{Introducción}

La insuficiencia cardiaca (IC) es unos de los principales problemas de la salud pública. Relativamente infrecuente antes de los 45 años, su incidencia casi se duplica con el paso de cada década de vida ${ }^{1}$. El envejecimiento general de la población y la prolongada supervivencia de los pacientes con cardiopatías isquémicas e hipertensión arterial han aumentado su prevalencia ${ }^{2}$, convirtiéndose en la causa más frecuente de hospitalización entre los pacientes mayores de 65 años ${ }^{1,3}$. La mortalidad entre los pacientes con IC es elevada (el $60 \%$ fallece a los 5 años de diagnóstico) y la atención a estos pacientes representa un 1-2\% del presupuesto total destinado a la atención sanitaria en los países industrializados. La mayor parte de este gasto se debe a los episodios de rehospitalización, ya que entre el 29 y el $47 \%$ de los pacientes con IC vuelve a ser ingresado a los 3-6 meses tras un primer ingreso por IC ${ }^{3}$. En España, la mitad de los pacientes hospitalizados debido a IC han sido ingresados anteriormente por la misma razón al menos una vez ${ }^{4}$.

Las causas de reingreso por IC son diversas ${ }^{5,6}$, pero las más importantes son la falta de cumplimiento del régimen terapéutico prescrito, la prescripción de un tratamiento inadecuado, la falta de apoyo social o el seguimiento inadecuado tras el alta hospitalaria ${ }^{7}$. Muchas de las crisis que requieren reingreso podrían evitarse. En concreto, numerosos estudios muestran que unos programas multidisciplinarios adecuados, 
de índole fundamentalmente educativa, pueden reducir de forma significativa el número, la duración y el coste de los reingresos e incluso la tasa de mortalidad entre los pacientes con $\mathrm{IC}^{8-22}$. En un metaanálisis reciente se recopilaron 27 estudios aleatorizados y 27 sin aleatorizar que evaluaban la eficacia de distintos programas de atención médica integral en pacientes ancianos y con insuficiencia cardiaca ${ }^{23}$. De forma resumida, según los datos provenientes de 10 estudios aleatorizados, las intervenciones redujeron el desenlace combinado de reingreso o fallecimiento en un $18 \%$. Dichas intervenciones estaban diseñadas y adaptadas a las posibilidades y los recursos locales, con una amplia variedad de contenidos y de profesionales implicados. La cuestión que surge, por lo tanto, es la siguiente: ¿cuál es el mejor modo de utilizar los recursos locales? La prestación de atención multidisciplinaria en el domicilio del paciente no está al alcance de muchos centros, pero limitar los esfuerzos para reducir los reingresos a las actuaciones en los consultorios o en medidas puramente educativas puede resultar ineficaz.

En este artículo presentamos los resultados de una evaluación, en términos de la reducción de la tasa de reingresos y muertes al año, de un programa básicamente educativo y a corto plazo llevado a cabo en los domicilios de los pacientes a través de una unidad de hospitalización domiciliaria ya existente, que está disponible en muchos hospitales españoles.

\section{Métodos}

Diseño del estudio

Se trata de un ensayo clínico controlado y aleatorizado llevado a cabo con pacientes de un hospital de atención terciaria y aprobado por el Comité Ético de Investigación Clínica de Galicia.

\section{Pacientes}

Eran pacientes incluibles en el estudio los dados de alta, tras un ingreso por IC, entre febrero de 2001 y junio de 2002, procedentes de las unidades de medicina interna, cardiología y de la unidad de corta estancia médica. Fueron invitados a participar los pacientes que vivían en el área cubierta por la unidad de hospitalización domiciliaria colaboradora, tenían suficiente apoyo familiar y no presentaban deficiencias cognitivas graves, enfermedades psiquiátricas avanzadas, enfermedad terminal no cardiológica y enfermedad pulmonar obstructiva crónica. Toda la información basal se obtuvo antes del alta del paciente y antes de ser asignados al grupo, incluida la causa más probable de la descompensación, según el médico responsable. Cuando ésta fue un mal cumplimiento de las recomendaciones de tratamiento, los pacientes se clasificaron como «no adherentes».

\section{Aleatorización y tamaño de la muestra}

Tras el alta hospitalaria, los pacientes que participaban en el estudio dieron su consentimiento informado por escrito y fueron asignados de forma aleatoria al grupo de control o al programa (grupo de intervención) mediante sobres cerrados y preparados en el Instituto Universitario de Ciencias de la Salud. El proceso de aleatorización se estratificó por servicio participante (medicina interna, cardiología y corta estancia médica). La secuencia de aleatorización se mantuvo oculta hasta asignar las intervenciones. Suponiendo la ausencia de pérdidas durante el seguimiento y una tasa de sucesos al año del $40 \%$ en el grupo de control, un tamaño de muestra de 279 personas permite la detección de una reducción del riesgo relativo del $40 \%$ mediante una prueba bilateral, con un valor $\alpha=0,05$ y $\beta=0,2$ (potencia del $80 \%$ ). El grupo definitivo de estudio, seleccionado entre febrero de 2001 y junio de 2002, incluye a 137 pacientes asignados al grupo de intervención y a 142 como controles. Sólo dos de los 281 pacientes invitados a participar rehusaron hacerlo. Ninguno de los miembros del equipo que asistió a estos pacientes durante los siguientes 12 meses, salvo los pertenecientes a la unidad de hospitalización domiciliaria, sabía si los pacientes pertenecían al grupo de intervención o de control.

\section{Intervención}

En el período comprendido entre la salida del hospital y la estabilización (hasta 15 días después), los pacientes asignados al grupo de intervención fueron atendidos en sus domicilios por personal médico y de enfermería pertenecientes a la unidad de hospitalización domiciliaria. Este servicio no es exclusivo para pacientes con IC. Estas unidades están destinadas a reducir la hospitalización en una variedad de condiciones médicas y quirúrgicas. Algunos de los servicios de atención sanitaria proporcionados son: 
exámenes clínicos, pruebas y análisis adicionales cuando son necesarios, y ajuste del régimen terapéutico atendiendo al progreso del paciente. Además, el personal de enfermería visitó a cada paciente 2, 5 y 10 días después del alta para administrar el programa educativo, que consistía en enseñar a los pacientes y familiares datos básicos sobre la IC y su tratamiento (síntomas, modo de vida, régimen alimentario, terapia). Cada paciente también recibió un manual impreso con la misma información, y un número de teléfono al que podía llamar para resolver sus dudas. Cada una de estas visitas tenía una duración aproximada de una hora. Aunque existía la posibilidad, no se hizo hincapié especial en que los pacientes utilizasen el servicio telefónico, y apenas hicieron uso de éste transcurridos 15 días después de la aleatorización. Por otra parte, se hizo hincapié en el cumplimiento (adhesión) del régimen terapéutico, el control del peso y la presión arterial, la ingestión de líquidos y la manera de reconocer y responder adecuadamente ante los signos de descompensación. Cuando fue necesario, el médico realizó ajustes en el tratamiento durante la primera semana. La parte más extensa de la intervención fue realizada por el personal de enfermería.

\section{Período de seguimiento}

Después de la intervención, los pacientes asignados a este grupo fueron remitidos a su médico de atención primaria para recibir atención adicional, al igual que se hizo con los pacientes del grupo de control tras el alta hospitalaria. Todos los pacientes recibieron un seguimiento de 12 meses tras el alta o hasta su muerte, si ésta se produjo durante dicho período. El seguimiento se realizó por medio de entrevistas telefónicas llevadas a cabo 3, 6 y 12 meses después del ingreso, y se complementaron con el examen de los registros clínicos de los pacientes participantes en el estudio.

\section{Medidas de los efectos y análisis estadístico}

La variable primaria empleada para evaluar la eficacia del programa fue la incidencia acumulada y combinada de reingreso o muerte 6 y 12 meses después del alta hospitalaria. Las variables secundarias evaluadas fueron las tasas acumuladas de reingreso y mortalidad por separado, la duración del reingreso y el uso de los servicios de urgencia durante los primeros 6 meses. Los ingresos programados no se consideraron sucesos. Se comparó a los pacientes de los grupos de intervención y control respecto a la variable primaria, no sólo en el grupo total de estudio, sino también en subgrupos definidos por la edad (menor o mayor de 75 años), sexo, fracción de eyección (menor o mayor del 40\%), etiología (isquémica u otra) y las causas de la descompensación que condujeron al ingreso original (incumplimiento del régimen de tratamiento frente a cualquier otra causa). Todas estas variables fueron claramente definidas en el protocolo. En la propuesta original no se definió ningún análisis de los subgrupos. La significación estadística de las diferencias entre los grupos se calculó mediante las pruebas de la $\chi^{2}$ y la t de Student para variables discretas y continuas, respectivamente, o mediante la prueba del orden logarítmico para la comparación de las curvas de supervivencia de Kaplan-Meier. Todos los análisis fueron realizados por intención de tratar.

\section{Resultados}

De los 279 pacientes que participaron en el estudio, $207(74,2 \%)$ procedían del servicio de medicina interna, $70(25,1 \%)$ del servicio de estancias cortas y $2(0,7 \%)$ del servicio de cardiología. De los 137 asignados al grupo de intervención, el personal de la unidad de hospitalización domiciliaria que administró el programa consideró que $127(92,7 \%)$ se habían mostrado cooperativos. No se produjo ninguna pérdida en el seguimiento.

Al inicio del estudio, los grupos de intervención y control no presentaban diferencias significativas en ninguna de las variables consideradas de interés (tabla 1). En el grupo de estudio completo, con 279 pacientes, la media de edad fue de 75,8 años; la etiología más frecuente fue la cardiopatía hipertensiva (43\%); la causa más frecuente de descompensación que motivó el ingreso índice fue el incumplimiento del régimen terapéutico $(36,2 \%)$ y la fracción de eyección media fue del 49,6\%. A la mayoría de los pacientes $(84 \%)$ se les prescribió inhibidores de la enzima de conversión de la angiotensina (IECA) o antagonistas de los receptores de la angiotensina II tras el alta hospitalaria; sólo al 12\% se le prescribió bloqueadores beta. 
Tabla 1. Características de los grupos de intervención y de control tras la aleatorización

\begin{tabular}{|c|c|c|}
\hline & Programa $(n=137)$ & Control $(n=142)$ \\
\hline Edad (años), media \pm DE & $75,3 \pm 11,1$ & $76,3 \pm 9,4$ \\
\hline Mujeres & 61,3 & 59,9 \\
\hline Vive solo & 14,6 & 12,0 \\
\hline Educación primaria o inferior & 86,8 & 90,9 \\
\hline Primer ingreso debido a IC & 54,0 & 53,5 \\
\hline \multicolumn{3}{|l|}{ Antecedentes } \\
\hline Hipertensión arterial & 66,4 & 69,0 \\
\hline Arritmia & 46,7 & 46,5 \\
\hline Diabetes & 35,8 & 35,2 \\
\hline Infarto de miocardio agudo previo & 19,7 & 17,6 \\
\hline Insuficiencia renal & 25,5 & 22,5 \\
\hline \multicolumn{3}{|l|}{ Etiología } \\
\hline Hipertensiva & 43,1 & 43,0 \\
\hline Cardiopatía isquémica & 29,2 & 31,7 \\
\hline \multicolumn{3}{|l|}{ Causa inmediata de ingreso* } \\
\hline Incumplimiento del tratamiento & 32,8 & 39,4 \\
\hline Arritmia & 32,1 & 36,6 \\
\hline Infecciones & 29,2 & 33,8 \\
\hline Otras/desconocida & 13,9 & 7,7 \\
\hline Fibrilación auricular & 53,3 & 52,1 \\
\hline Fracción de eyección $(\%)$, media \pm DE & $50,9 \pm 16,6(n=130)$ & $48,3 \pm 17,6 \quad(n=124)$ \\
\hline \multicolumn{3}{|l|}{ Tratamiento en el alta } \\
\hline IECA/ARA-II & 85,4 & 82,4 \\
\hline Bloqueadores beta & 13,1 & 10,6 \\
\hline Anticoagulantes & 39,4 & 37,3 \\
\hline
\end{tabular}

ARA-II: antagonistas de los receptores de la angiotensina II; DE: desviación estándar; IC: insuficiencia cardiaca; IECA: inhibidores de la enzima de conversión de la angiotensina.

* Más de uno posible (por lo tanto, los porcentajes no suman 100).

Valores expresados en porcentajes, a menos que se indique lo contrario.

Durante el período de 12 meses de seguimiento, los grupos del programa y de control se comportaron de forma muy similar en cuanto a la incidencia acumulada de reingresos y muertes (tabla 2). Aunque la tasa combinada de reingreso y mortalidad fue mayor en el grupo de control que en el de intervención, al igual que las tasas de reingresos y de mortalidad por separado (salvo en lo que respecta a la tasa de mortalidad al año), en ningún caso la diferencia resultó estadísticamente significativa (tabla 2; fig. 1).

Tabla 2. Incidencia acumulada de desenlaces clínicos 6 y 12 meses después del alta hospitalaria tras insuficiencia cardiaca

\begin{tabular}{|c|c|c|c|c|c|c|}
\hline \multirow[b]{2}{*}{ Suceso } & \multicolumn{6}{|c|}{ Grupo } \\
\hline & Duración de seguimiento & $\begin{array}{l}\text { Programa } \\
(\mathrm{n}=137)\end{array}$ & $\begin{array}{l}\text { Control } \\
(\mathrm{n}=142)\end{array}$ & Riesgo relativo $^{\mathrm{b}}$ & $\begin{array}{c}\text { Intervalo de } \\
\text { confianza del } 95 \%\end{array}$ & $\mathrm{p}$ \\
\hline \multirow[t]{2}{*}{ Reingreso ${ }^{a}$ o muerte } & 6 meses & 32,1 & 38,0 & 0,85 & $0,61-1,16$ & 0,318 \\
\hline & 1 año & 45,3 & 52,8 & 0,86 & $0,67-1,09$ & 0,232 \\
\hline \multirow[t]{2}{*}{ Reingreso $^{\mathrm{a}}$} & 6 meses & 30,7 & 35,9 & 0,85 & $0,61-1,19$ & 0,376 \\
\hline & 1 año & 43,1 & 50,0 & 0,86 & $0,67-1,11$ & 0,280 \\
\hline \multirow[t]{2}{*}{ Muerte } & 6 meses & 5,8 & 9,9 & 0,59 & $0,26-1,37$ & 0,268 \\
\hline & 1 año & 16,1 & 14,8 & 1,08 & $0,63-1,88$ & 0,769 \\
\hline
\end{tabular}

axclusión de ingresos programados.

${ }^{\mathrm{b}}$ Incidencia programa/control. 


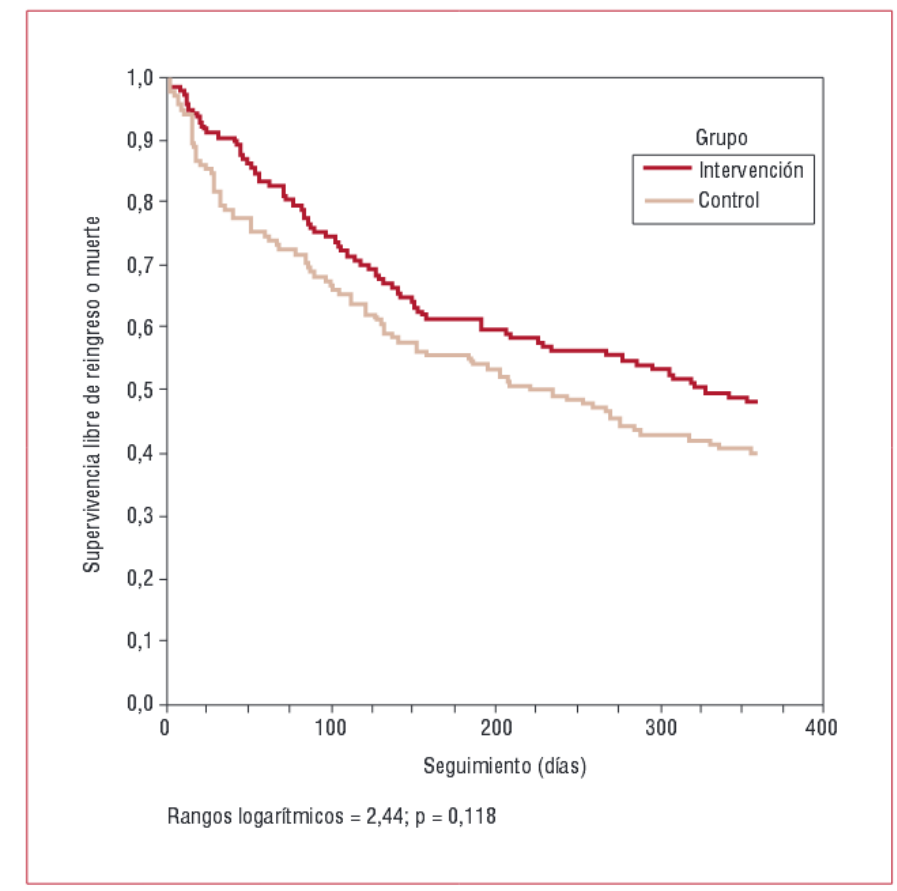

Fig. 1. Supervivencia libre de reingreso o muerte en los grupos de intervención y de control

No sólo el número, sino también las características de los reingresos fueron similares en ambos grupos. En el grupo de intervención, 59 pacientes fueron reingresados de forma no programada en un total de 125 ocasiones (frente a 71 pacientes con 118 episodios de reingreso en el grupo de control) y 39 accedieron en 59 ocasiones a urgencias hospitalarias en los primeros 6 meses (frente a 42 pacientes y 57 ocasiones en el grupo de control); por cada 100 pacientes del grupo de intervención seguidos durante un año se produjeron 91 reingresos sin programar, 43 ingresos en servicio de urgencias en los primeros 6 meses y 16 muertes (en comparación, se produjeron 83 reingresos sin planificar, 40 ingresos en urgencias y 15 muertes en el grupo de control). Ninguna de las diferencias entre los grupos de intervención y de control en cuanto a las características anteriores es estadísticamente significativa (tabla 3). 
Tabla 3. Desenlaces durante el seguimiento de 12 meses de pacientes dados de alta tras insuficiencia cardiaca

\begin{tabular}{|c|c|c|}
\hline & \multicolumn{2}{|c|}{ Grupo } \\
\hline & Programa & Control \\
\hline Número de reingresos & 146 & 137 \\
\hline Programados & 21 & 19 \\
\hline Sin programar & 125 & 118 \\
\hline Insuficiencia cardiaca & 55 & 57 \\
\hline Alguna otra causa cardiovascular & 25 & 21 \\
\hline Causa no cardiovascular & 45 & 40 \\
\hline Duración (días) & $8,4 \pm 7,7$ & $9,6 \pm 13,0$ \\
\hline Programada & $7,3 \pm 10,3$ & $6,5 \pm 14,0$ \\
\hline Sin programar & $8,6 \pm 7,2$ & $10,1 \pm 12,9$ \\
\hline Insuficiencia cardiaca & $8,5 \pm 6,4$ & $8,4 \pm 11,6$ \\
\hline Alguna otra causa cardiovascular & $6,1 \pm 4,6$ & $10,9 \pm 10,7$ \\
\hline Causa no cardiovascular & $10,0 \pm 8,6$ & $12,0 \pm 15,4$ \\
\hline \multicolumn{3}{|l|}{ Número de ingresos } \\
\hline Servicios de urgencias* & 59 & 57 \\
\hline Insuficiencia cardiaca & 7 & 10 \\
\hline Alguna otra causa cardiovascular & 5 & 5 \\
\hline Otras causas & 27 & 35 \\
\hline Mal definido & 18 & 6 \\
\hline Muertes & 22 & 21 \\
\hline Causa cardiovascular & 16 & 18 \\
\hline Causa no cardiovascular & 6 & 3 \\
\hline
\end{tabular}

* Durante los primeros 6 meses del seguimiento.

Los valores expresan el número o la media \pm desviación estándar

Tabla 4. Incidencia acumulada ${ }^{\mathrm{a}}$ los 12 meses de reingresos ${ }^{\mathrm{b}}$ o muertes en los subgrupos de los grupos de intervención y de control

\begin{tabular}{|c|c|c|c|c|c|}
\hline & \multicolumn{2}{|c|}{ Grupo } & \multirow[b]{2}{*}{ Riesgo relativo $(\%)^{\mathrm{c}}$} & \multirow[b]{2}{*}{ IC del 95\% } & \multirow[b]{2}{*}{$\mathrm{p}$} \\
\hline & Programa & Control & & & \\
\hline \multicolumn{6}{|l|}{ Edad (años) } \\
\hline $\begin{array}{l}<75 \\
\geq 75\end{array}$ & $\begin{array}{l}40,6(26 / 64) \\
49,3(36 / 73)\end{array}$ & $\begin{array}{l}45,3(24 / 53) \\
57,3(51 / 89)\end{array}$ & $\begin{array}{l}0,90 \\
0,86\end{array}$ & $\begin{array}{l}0,59-1,36 \\
0,64-1,15\end{array}$ & $\begin{array}{l}0,708 \\
0,344\end{array}$ \\
\hline \multicolumn{6}{|l|}{ Sexo } \\
\hline $\begin{array}{l}\text { Varones } \\
\text { Mujeres }\end{array}$ & $\begin{array}{l}49,1(26 / 53) \\
42,9(36 / 84)\end{array}$ & $\begin{array}{l}50,9(29 / 57) \\
54,1(46 / 85)\end{array}$ & $\begin{array}{l}0,96 \\
0,79\end{array}$ & $\begin{array}{l}0,66-1,40 \\
0,58-1,09\end{array}$ & $\begin{array}{l}1,000 \\
0,167\end{array}$ \\
\hline \multicolumn{6}{|l|}{ Etiología } \\
\hline $\begin{array}{l}\text { Isquémica } \\
\text { Otra }\end{array}$ & $\begin{array}{l}57,5(23 / 40) \\
40,2(39 / 97)\end{array}$ & $\begin{array}{l}56,5(26 / 46) \\
51,0(49 / 96)\end{array}$ & $\begin{array}{l}1,02 \\
0,79\end{array}$ & $\begin{array}{l}0,70-1,47 \\
0,58-1,08\end{array}$ & $\begin{array}{l}1,000 \\
0,149\end{array}$ \\
\hline \multicolumn{6}{|c|}{ Fracción de eyección } \\
\hline $\begin{array}{l}\leq 40 \% \\
>40 \%\end{array}$ & $\begin{array}{l}622(23 / 37) \\
41,9(39 / 93)\end{array}$ & $\begin{array}{l}76,2(32 / 42) \\
52,4(43 / 82)\end{array}$ & $\begin{array}{l}0,82 \\
0,80\end{array}$ & $\begin{array}{l}0,60-1,10 \\
0,58-1,10\end{array}$ & $\begin{array}{l}0,223 \\
0,175\end{array}$ \\
\hline \multicolumn{6}{|c|}{ Causas inmediatas, incluye incumplimiento del tratamiento } \\
\hline $\begin{array}{l}\text { Sí } \\
\text { No }\end{array}$ & $\begin{array}{l}35,6(16 / 45) \\
50,0(46 / 92)\end{array}$ & $\begin{array}{l}60,7(34 / 56) \\
47,7(41 / 86)\end{array}$ & $\begin{array}{l}0,59 \\
1,05\end{array}$ & $\begin{array}{l}0,37-0,91 \\
0,78-1,42\end{array}$ & $\begin{array}{l}0,016 \\
0,766\end{array}$ \\
\hline
\end{tabular}

IC: intervalo de confianza.

${ }^{a}$ Expresado como porcentajes y, en paréntesis, la razón del número de pacientes afectados al número en el grupo.

${ }^{\mathrm{b}}$ Excluye los ingresos programados.

${ }^{\mathrm{c}}$ Incidencia programa/control. 

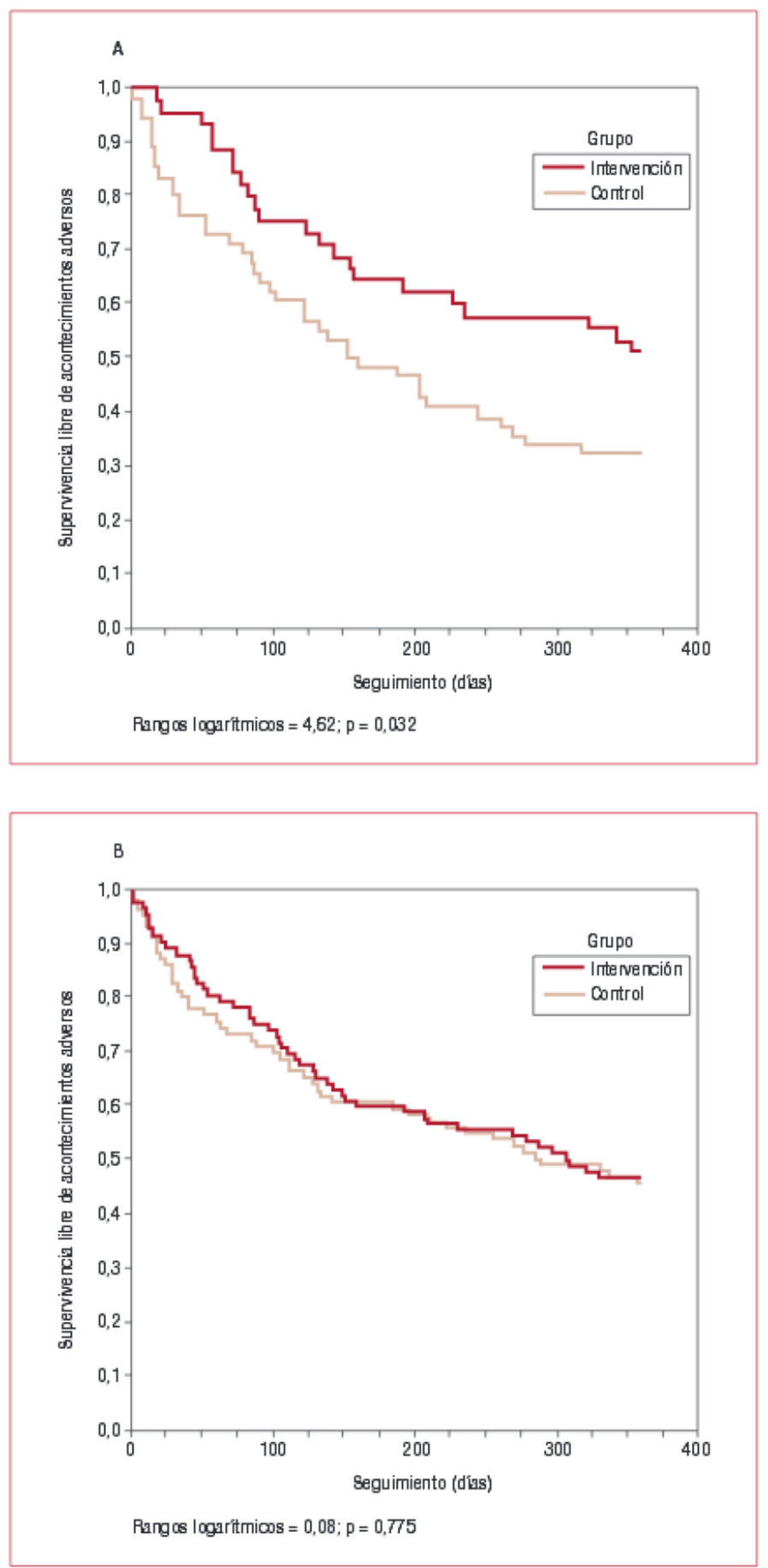

Fig. 2. Supervivencia libre de reingreso o muerte en subgrupos de los grupos de intervención y de control definidos como: pacientes cuyo ingreso inicial fue debido en parte al incumplimiento del régimen terapéutico (A), y otros (B) 


\section{Discusión}

Este ensayo clínico muestra que esta intervención breve en el tiempo y de índole fundamentalmente educativa tiene, en el mejor de los casos, un efecto muy moderado sobre la reducción de reingresos cuando se administra a todos los pacientes con IC dados de alta del hospital. La magnitud del efecto en el grupo en su totalidad, que ha resultado no significativo en este estudio, es muy similar al documentado en un metaanálisis reciente ${ }^{23}$. No obstante, el subgrupo de pacientes ingresados por incumplir el régimen terapéutico puede beneficiarse enormemente de este tipo de intervención.

La IC es un síndrome crónico con elevadas tasas de morbimortalidad que no parecen haberse reducido de forma inequívoca y de modo sustancial con los avances terapéuticos de las últimas décadas. Unos autocuidados adecuados pueden reducir el riesgo de episodios que requieran el reingreso, pero su adquisición y aplicación sistemática por parte de los pacientes con IC se ven obstaculizadas por el hecho de que estos pacientes son generalmente de edad avanzada. Para aumentar la proporción de pacientes con IC conscientes de la necesidad y la importancia de dichas prácticas de cuidados personales, numerosos grupos de investigación han diseñado programas de diversa intensidad: desde una sencilla llamada telefónica por parte del personal de enfermería hasta un seguimiento intensivo por parte de un equipo multidisciplinario. En general, los programas basados en contactos periódicos han funcionado mejor que los realizados en una única intervención; los que combinan la atención clínica con la educativa también muestran mejores resultados que los de cariz exclusivamente educativo; y por último, los programas realizados en el domicilio del paciente son más exitosos que los que requieren la asistencia de éste a una clínica o consultorio $^{8-22}$. En 2 revisiones sistemáticas recientes se ofrecen puntos de vista distintos acerca del papel del tipo de intervención. Mientras que una señala que éste es importante ${ }^{24}$, en la otra, «se observaron (resultados) independientemente del tipo de prestación sanitaria proporcionada en los programas de cuidado integral de la enfermedad» ${ }^{23}$. Después de estas publicaciones, se han publicado al menos otros dos estudios aleatorizados y de grandes dimensiones. Uno de ellos no muestra efectos en la rehospitalización por cualquier causa ${ }^{25}$, mientras que en el otro, que incluía a más de 1.000 pacientes, se observó un beneficio en la mortalidad (aunque no aporta las reducciones del riesgo relativo) sin reducción en los $\operatorname{costes}^{26}$. El artículo editorial que acompaña a este último estudio ${ }^{27}$ hace hincapié nuevamente en la importancia del tipo de intervención. Nuestro estudio indica que los pacientes que más se pueden beneficiar de una intervención educativa son los que no cumplen de forma sistemática las recomendaciones de cuidados sanitarios indicadas. Otro tipo de intervenciones podría beneficiar más a otro tipo de pacientes, lo que subraya la necesidad de seleccionar de forma metódica a los pacientes que participan en estos programas. El interés de nuestro estudio fue evaluar un programa diseñado para integrarse dentro de las capacidades de la unidad de hospitalización domiciliaria de nuestro centro. Por consiguiente, la intervención es fácilmente transferible a los muchos otros hospitales españoles que cuentan con este tipo de unidad, a fin de permitir el alta de pacientes que aún necesitan supervisión médica de ámbito hospitalario, pero que no requieren la utilización intensiva de servicios de enfermería ni acceso inmediato a otras instalaciones del hospital. Aunque el programa fue muy corto y no incluía contactos periódicos con los pacientes elegidos, se basaba en la asistencia del paciente en su hogar y una combinación de atención sanitaria y educativa, con hincapié en esta última. Por la propia naturaleza de la intervención, los pacientes y el personal de la unidad de hospitalización domiciliaria que participaron en este estudio no lo hicieron «a ciegas» respecto al tratamiento, pero el personal que atendía a los pacientes en otros servicios desconocía si éstos pertenecían al programa o al grupo de control. Por consiguiente, la asignación de desenlaces se realizó a ciegas. No se produjeron abandonos, y las características al inicio de los grupos del programa y de control parecen reflejar una aleatorización eficaz.

A diferencia de algunos otros estudios ${ }^{3,9,10}$, el nuestro no se limitó a pacientes con un alto riesgo de reingreso (pacientes con etiología isquémica, disfunción sistólica, antecedentes de ingresos múltiples por IC en el año anterior, etc.). Por el contrario, el grupo de estudio constituía una muestra bastante representativa de los pacientes dados de alta hospitalaria tras la IC, y su limitación principal en este sentido era la exclusión de pacientes con enfermedad pulmonar obstructiva crónica. Una proporción elevada de pacientes fue tratada con IECA en el momento del alta, lo que dejaba pocas posibilidades de añadir mejoras. Sin embargo, en muchos pacientes no se había introducido los bloqueadores beta debido a la duración de la intervención. La mayor parte del efecto debe atribuirse, por consiguiente, a los componentes educativos de la intervención.

En términos generales, creemos que nuestra intervención tuvo lugar durante un corto período en el que el personal médico y de enfermería se centró en la optimización del tratamiento y de los temas de educación sanitaria. Los participantes en el programa apenas recurrieron al servicio adicional de consulta telefónica, quizá debido a que el personal de la unidad domiciliaria no recalcó esta posibilidad tanto como en otros programas con servicios telefónicos similares ${ }^{8-11,19,20}$. Respecto al diseño de la intervención, los programas más exitosos combinan la atención clínica periódica en el hogar del paciente (evaluación de la condición clínica y ajustes en el tratamiento) con la educación sobre cuidados personales ${ }^{22}$. Los 
programas que sólo proporcionan educación son menos costosos, pero también mucho menos eficaces $^{11,13}$, mientras que los programas que exigen la asistencia del paciente a un consultorio tampoco muestran buenos resultados ${ }^{17}$.

Un estudio español publicado recientemente ${ }^{28}$ documenta reducciones del $90 \%$ en los reingresos, del $84,2 \%$ en la asistencia a los servicios de urgencia y del 82,4\% en las muertes. Estas diferencias tan enormes con nuestros resultados (con reducciones que oscilan alrededor del 15\%) no se deben al tipo de intervención, porque era similar a la nuestra aunque más corta (sólo una visita de 2 horas sin refuerzo). Una posible explicación podría ser las diferencias en el tipo de paciente. En el citado estudio, los pacientes presentan un nivel cultural más elevado (el 82\% tenía un nivel de estudios medio-alto, mientras que en el nuestro el $88 \%$ de los participantes sólo tenía estudios primarios) y un peor pronóstico (la fracción de eyección era inferior y había más cardiopatías isquémicas) que nuestros pacientes, lo que se reflejó en la elevada mortalidad del grupo de control (11/36 [30,5\%] en 6 meses) y que podría haber causado que la intervención fuese más eficaz. Los desequilibrios entre grupos en un estudio tan pequeño como éste (70 pacientes, en comparación con los 279 en el nuestro) también pueden tener un cierto papel en la explicación de las diferencias.

\section{Limitaciones}

Algunas de las limitaciones de este estudio son la corta duración de la intervención y la ausencia de mediciones de los ajustes posteriores de tratamiento. Tampoco se midieron los efectos de la intervención a medio plazo (p. ej., las mejoras en el cumplimiento del tratamiento entre los pacientes «no adherentes»). Por último, la información procedente de los análisis de subgrupos debe interpretarse con cautela porque se obtuvo mediante los análisis post hoc.

\section{Conclusiones}

Los recursos de nuestra unidad de hospitalización domiciliaria resultaron suficientes para la administración de un programa, fundamentalmente educativo y de corta duración, a pacientes dados de alta tras un ingreso hospitalario por insuficiencia cardiaca. La intervención no redujo la incidencia de reingresos ni de muertes entre estos pacientes (sólo se produjo una reducción del riesgo relativo del $14,3 \%$, sin significación estadística). Un análisis post hoc indica que todo el beneficio podría recaer sobre los pacientes con IC cuya hospitalización fue en parte debida al incumplimiento del régimen terapéutico. La aplicación eficaz de tales recursos precisaría formular preguntas específicas a los pacientes con IC hospitalizados con el fin de identificar a los que tienen un nivel bajo de cumplimiento. Asimismo, su extensión a todos los pacientes con IC con miras a prevenir un primer ingreso por IC requeriría la identificación de los factores predictivos del incumplimiento. Puesto que el tipo de unidad que administró el programa es habitual en los hospitales españoles, el programa puede ser fácilmente transferible a estos otros centros.

\section{Agradecimientos}

Los autores agradecen a Ignacio Castro San Martín, del Instituto Universitario de Ciencias de la Salud de La Universidad de A Coruña, por la gestión de los datos y su asistencia con la programación informática, y a Juan Escudero Pereira, jefe del Departamento de Gestión del Complejo Hospitalario Universitario Juan Canalejo, por su asistencia para identificar a candidatos para el estudio.

Guillermo Mourenza Díaz e Isabel Bermúdez Ares (diplomados universitarios en Enfermería) fueron responsables de administrar la intervención.

Luciano Doval Domínguez y Victoria Martínez Lago (médicos) participaron en la selección de pacientes.

\section{Bibliografía}

1.McMurray JJ, Stewart S. Epidemiology, aetiology, and prognosis of heart failure. Heart. 2000;83:596-602.

2.Cowie MR, Mosterd A, Wood DA, Deckers JW, Poole- Wilson PA, Sutton CG, et al. The epidemiology of heart failure. Eur Heart J. 1997;18:208-25.

3.Rich MW, Beckham V, Wittenberg C, Leven CL, Freedland KE, Carney RM. A multidisciplinary intervention to prevent the readmission of elderly patients with congestive heart failure. N Engl J Med. 1995;333:1190-5.

4.García Castelo A, Muñiz J, Sesma P, Castro Beiras A, on behalf of the INCARGAL study group. Utilización de recursos diagnósticos y terapéuticos en pacientes ingresados por insuficiencia cardiaca: influencia del servicio de ingreso (estudio INCARGAL). Rev Esp Cardiol. 2003;56:49-56. 
5.Ghali JK, Kadakia S, Cooper R, Ferlinz J. Precipitating factors leading to decompensation of heart failure. Arch Intern Med. 1988;148:2013-6.

6.Opasich C, Febo O, Riccardi G, Traversi E, Forni G, Pinna GM, et al. Concomitant factors of decompensation in chronic heart failure. Am J Cardiol. 1996;78:354-7.

7.West JA, Miller NH, Parker KM, Senneca D, Ghandour G, Clark M, et al. A comprehensive management system for heart failure improves clinical outcomes and reduces medical resource utilization. Am J Cardiol. 1997;79:5863.

8.Blue L, Lang E, McMurray J, Davie AP, McDonagh TA, Murdoch DR, et al. Randomised controlled trial of specialist nurse intervention in heart failure. BMJ. 2001;323:715-8.

9.Naylor MD, Brooten D, Campbell R, Jacobsen BS, Mezey MD, Pauly MV, et al. Comprehensive discharge planning and home follow-up of hospitalized elders: a randomized clinical trial. JAMA. 1999;281:613-20.

10.Kasper EK, Gerstenblith G, Hefter G, Van Anden E, Brinker JA, Thiermann DR, et al. A randomized trial of the efficacy of multidisciplinary care in heart failure outpatients at high risk of hospital readmission. J Am Coll Cardiol. 2002;39:471-80.

11.Krumholz HM, Amatruda J, Smith GL, Mattera JA, Roumanis SA, Radford MJ, et al. Randomized trial of an education and support intervention to prevent readmission of patients with heart failure. J Am Coll Cardiol. 2002;39:83-9.

12.Weinberger M, Oddone EZ, Henderson WG. Does increased access to primary care reduce hospital readmissions? N Engl J Med. 1996;334:1441-7.

13.Jaarsma T, Halfens R, Huijer Abu-Saad H, Dracup K, Van Ree GJ, Stappers J. Effects of education and support on self-care and resource utilization in patients with heart failure. Eur Heart J. 1999;20:673-82.

14.Jerant AF, Azari R, Nesbitt TS. Reducing the cost of frequent hospital admissions for congestive heart failure. Med Care. 2001; 39:1234-45.

15.Varma S, McElnay JC, Hughes CM, Passmore AP, Varma M. Pharmaceutical care of patients with congestive heart failure: interventions and outcomes. Pharmacotherapy. 1999;19:860- 9.

16.Doughty RN, Wright SP, Pearl A, Walsh HJ, Muncaster S, Whalley GA, et al. Randomized controlled trial of integrated heart failure management. Eur Heart J. 2002;23:139-46.

17.Ekman I, Andersson B, Ehnfors M, Matejka G, Persson B, Fagerberg B. Feasibility of a nurse-monitored outpatient-care programme for elderly patients with moderate-to-severe chronic heart failure. Eur Heart J. 1998; 19:1254-60.

18.Gattis WA, Hasselblad V, Whellan DJ, O Connor CM. Reduction in heart failure events by the addition of a clinical pharmacist to the heart failure management team. Arch Intern Med. 1999;159: 1939-45.

19.Cline CM.J, Israelsson BY.A, Willenheimer RB, Broms K, Erhardt LR. Cost-effective management programme for heart failure reduces hospitalisation. Heart. 1998;80:442-6.

20.Stewart S, Marley JE, Horowitz JD. Effects of a multidisciplinary, home- based intervention on unplanned readmissions and survival among patients with chronic congestive heart failure: a randomised controlled study. Lancet. 1999;354:1077-83

21.Serxner S, Miyaji M, Jeffords J. Congestive heart failure disease management study: a patient education intervention. Congest Heart Fail. 1998;4:23-8.

22.Rich MW. Heart failure disease management: A critical review. J Card Fail. 1999;5:64-75.

23.Gonseth J, Guallar-Castellón P, Banegas JR, Rodríguez-Artalejo F. The effectiveness of disease management programmes in reducing hospital re-admission in older patients with heart failure: a systematic review and metaanalysis of Publisher reports. Eur Heart J. 2004;25:1570-95.

24.McAlister FA, Stewart S, Ferrua S, McMurray JJ. Multidisciplinary strategies for the management of heart failure patients at high risk for admission: a systematic review of randomized trials. J Am Coll Cardiol. 2004;44:810-9.

25.DeBusk RF, Miller NH, Parker KM, Bandura A, Kraemer HC, Che.r, et al. Care management for low risk patients with heart failure. A randomized, controlled trial. Ann Intern Med. 2004;141: 606-13.

26.Galbreath AD, Krasuski RA, Smith B, Stajduhar KC, Kwan MD, Ellis R, et al. Long -term healthcare and cost outcomes of disease management in a large, randomized, community-based population with heart failure. Circulation. 2004;110:3518-26.

27.Fonarow GC. Heart failure disease management programs. Not a class effect. Circulation. 2004;110:3506-8.

28. Morcillo C, Valderas JM, Aguado O, Delás J, Sort D, Pujadas R, et al. Evaluación de una intervención domiciliaria en pacientes con insuficiencia cardiaca. Resultados de un estudio aleatorizado. Rev Esp Cardiol. 2005;58:618-25 